\title{
Concentrations of high density lipoprotein cholesterol, triglycerides, and total cholesterol in ischaemic heart disease
}

\author{
Stuart J Pocock, A Gerald Shaper, Andrew N Phillips
}

\begin{abstract}
Objective-To assess the roles of serum concentrations of total cholesterol, high density lipoprotein cholesterol, and triglycerides in predicting major ischaemic heart disease.

Design-Men recruited for the British regional heart study followed up for a mean of 7.5 years.

Setting-General practices in 24 British towns.

Patients-7735 Middle aged men.

End point-Predictive value of serum concentrations of lipids.

Measurements and main results - At initial screening serum concentrations of total cholesterol, high density lipoprotein cholesterol, and triglycerides were determined from non-fasting blood samples. Altogether $\mathbf{4 4 3}$ major ischaemic heart disease events (fatal and non-fatal) occurred during the study. Men in the highest fifth of the distribution of total cholesterol concentration $(\geqslant 7.2 \mathrm{mmol} / \mathrm{l})$ had 3.5 times the risk of ischaemic heart disease than did men in the lowest fifth $(<5.5 \mathrm{mmol} / \mathrm{l})$ after adjustment for high density lipoprotein cholesterol concentration and other risk factors. Men in the lowest fifth of high density lipoprotein cholesterol concentration $(<0.93 \mathrm{mmol} / \mathrm{l})$ had 2.0 times the risk of men in the highest fifth $(\geqslant 1.33 \mathrm{mmol} / \mathrm{l})$ after adjustment for total cholesterol concentration and other risk factors. Men in the highest fifth of triglyceride concentration ( $\geqslant 2.8 \mathrm{mmol} / \mathrm{l}$ ) had only 1.3 times the risk of those in the lowest fifth $(<1.08 \mathrm{mmol} / \mathrm{l})$ after adjustment for total cholesterol concentration and other risk factors; additional adjustment for high density lipoprotein cholesterol concentration made the association with ischaemic heart disease disappear.
\end{abstract}

Conclusions-Serum concentration of total cholesterol is the most important single blood lipid risk factor for ischaemic heart disease in men. High density lipoprotein cholesterol concentration is less important, and triglyceride concentrations do not have predictive importance once other risk factors have been taken into account.

\section{Introduction}

Several prospective studies have reported that men Department of Clinical Epidemiology and General Practice, Royal Free Hospital School of Medicine, London NW3 2PF

Stuart J Pocock, PHD, professor of medical statistics A Gerald Shaper, FRCP. professor of clinical epidemiology Andrew N Phillips, PHD, medical statistician

Correspondence to: Professor Pocock. the United States, which questioned the validity of adjusting for non-high density lipoprotein cholesterol concentration, ${ }^{5}$ and the increased number of cases of ischaemic heart disease in a further three years of follow up we re-examined the British regional heart study's data on high density lipoprotein cholesterol concentration.

High triglyceride concentrations are associated with an increased risk of ischaemic heart disease, but the aetiological and predictive relevance of triglyceride concentrations is uncertain as high concentrations are associated with high total cholesterol and lower high density lipoprotein cholesterol concentrations. Though serum triglyceride concentrations do not independently predict major ischaemic heart disease events, ' interest in them has increased recently, possibly because new treatments are available that considerably lower them. As authoritative bodies have provided recommendations for management, including drug treatment, based on the serum concentrations of total cholesterol, high density lipoprotein cholesterol, and triglycerides ${ }^{8}$ reappraisal of the relative importance of these lipids to the risk of major ischaemic heart disease is important. Prospective studies provide the most valid approach to this problem, and we report results of a large study of British middle aged men.

\section{Subjects and methods}

In the British regional heart study 7735 men aged $\mathbf{4 0}$ to 59 selected at random from the age-sex registers of general practices in 24 towns in England, Wales, and Scotland were examined. The criteria for selecting the towns, general practices, and subjects and the methods of collecting data and measuring serum lipid concentrations have been described..$^{10-13}$ Non-fasting blood samples were obtained between 0830 and 1830 . Triglyceride concentrations tend to increase during the day in non-fasting patients, ${ }^{14}$ and therefore each subject's value was standardised for hour of day. These concentrations were not measured in the first six towns so data were available for only 5675 men. Serum concentrations of total cholesterol and high density lipoprotein cholesterol were not measured in 45 and 315 men, respectively. All 7735 men who were examined from 1978 to 1980 were followed up for both morbidity and mortality for eight years. ${ }^{12}$ At the time of this report $99 \%$ of the men had been followed up for an average of $7 \cdot 5$ years. Definitions of fatal and non-fatal cases of major ischaemic heart disease have been documented."

The simultaneous contributions of several factors to the risk of major ischaemic heart disease were analysed by analysis of covariance and a multiple logistic model. Specifically, the adjusted rates shown in the figure were obtained by using such models with each factor in turn fitted in five intervals - that is, with four dummy variables - whereas other factors were fitted as continuous measurements. ${ }^{15}$

\section{Results}

During an average of 7.5 years of follow up 443 of the 7735 men screened experienced a major ischaemic 
heart disease event. The men with ischaemic heart disease had significantly higher mean concentrations of total cholesterol and triglycerides and a significantly lower mean concentration of high density lipoprotein cholesterol than the other men (table I). The mean difference in high density lipoprotein cholesterol concentration of $0.075 \mathrm{mmol} / \mathrm{l}$ was slightly greater than in our earlier data. ${ }^{6}$

\section{INTERRELATIONS AND ASSOCIATIONS}

Total cholesterol and high density lipoprotein cholesterol concentrations were not associated $(\mathrm{r}=-0 \cdot 04)$, whereas triglyceride concentrations had important associations with concentrations of both total cholesterol $(r=0.37)$ and high density lipoprotein cholesterol $(r=-0 \cdot 46)$. Cigarette smoking was associated with a significant decrease in high density lipoprotein cholesterol concentration: men who smoked 20 or more cigarettes a day had a mean concentration of $1 \cdot 12 \mathrm{mmol} / \mathrm{l}$ whereas men who had never smoked had a mean concentration of $1.18 \mathrm{mmol} / \mathrm{l} .^{13}$ Mean triglyceride concentrations were also slightly lower in cigarette smokers. Body mass index was significantly associated with concentrations of total cholesterol, high density lipoprotein cholesterol, and triglycerides $(\mathrm{r}=0 \cdot 17,-0 \cdot 25$, and $0 \cdot 30$, respectively).

\section{INDEPENDENCE OF EFFECTS}

The above interrelations and associations with other risk factors were taken into account in considering the independent predictive relevance of each lipid to the risk of ischaemic heart disease. Analysis of covariance (table II) showed how the mean differences between men who experienced a major ischaemic heart disease event during follow up-that is, men who became cases - and other men were affected by adjustment for one another and for other risk factors (age, systolic blood pressure, body mass index, and years of smoking). Analysis of high density lipoprotein cholesterol and total cholesterol concentrations showed a slight narrowing of their mean differences after adjustment, but both variables remained highly significant. An analysis of triglyceride and total cholesterol concentrations showed that the higher mean triglyceride concentration in men who became cases was accounted for by the association of triglyceride concentrations with total cholesterol concentration and other risk factors.

Multiple logistic regression was used to estimate the standardised relative odds - that is, the change in

TABLE I-Mean (SEM) serum concentrations of total cholesterol, high density lipoprotein (HDL) cholesterol, and triglycerides in men who became cases of ischaemic heart disease (IHD) and other men

\begin{tabular}{|c|c|c|c|c|c|}
\hline \multirow[b]{2}{*}{ Lipid } & \multicolumn{2}{|c|}{ IHD cases } & \multicolumn{2}{|c|}{ Other men } & \multirow[b]{2}{*}{ t Statistic } \\
\hline & No of men & Concentration & No of men & Concentration & \\
\hline $\begin{array}{l}\text { Total cholesterol }(\mathrm{mmol} / \mathrm{l}) \\
\text { HDL cholesterol }(\mathrm{mmol} / \mathrm{l}) \\
\text { Triglycerides }(\mathrm{mmol} / \mathrm{l})\end{array}$ & $\begin{array}{l}438 \\
417 \\
324\end{array}$ & $\begin{array}{l}6.766(0.055) \\
1.076(0.013) \\
2 \cdot 331(0.077)\end{array}$ & $\begin{array}{l}7252 \\
7003 \\
5351\end{array}$ & $\begin{array}{l}6.270(0.012) \\
1.151(0.003) \\
2.032(0.018)\end{array}$ & $\begin{array}{r}8 \cdot 81 \\
-5 \cdot 62 \\
3 \cdot 78\end{array}$ \\
\hline
\end{tabular}

TABLE II - Unadjusted and adjusted ${ }^{\star}$ mean differences in lipid concentrations between men who did and did not become cases of ischaemic heart disease during follow up and standardised relative odds (for high density lipoprotein cholesterol and total cholesterol, 413 cases and 6857 other men; for triglycerides and total cholesterol, 313 cases and 5200 other men)

\begin{tabular}{lcccc}
\hline & \multicolumn{3}{c}{ Mean differences } \\
\cline { 2 - 3 } Lipid & Unadjusted & $\begin{array}{c}\text { Adjusted }(95 \% \text { confidence } \\
\text { interval })\end{array}$ & $\begin{array}{c}\text { Standardised } \\
\text { relative odds } \dagger\end{array}$ & p Value \\
\hline HDL cholesterol & -0.075 & $-0.060(-0.086$ to -0.034$)$ & 1.30 & $<<0.0001$ \\
Total cholesterol & 0.495 & $0.461(0.358$ to 0.564$)$ & 1.54 & $<0.0001$ \\
Triglycerides & 0.299 & $0.009(-0.132$ to 0.150$)$ & 0.99 & 0.53 \\
Total cholesterol & 0.482 & $0.378(0.266$ to 0.490$)$ & 1.52 & $\ll 0.0001$
\end{tabular}

«Each part adjusted for each other and for age, systolic blood pressure, body mass index, and years of smoking. †Refers to a reduction of $1 S D$ in high density lipoprotein cholesterol and increases of $1 S D$ in total cholesterol and triglyceride concentration. odds of an ischaemic heart disease event for a change of $1 \mathrm{SD}$ in the risk factor-after adjustment for other risk factors. Table II shows that the standardised relative odds for both total cholesterol and high density lipoprotein cholesterol concentrations were highly significant, with total cholesterol concentration showing the stronger independent association with risk of ischaemic heart disease. The standardised relative odds for triglyceride concentrations were close to unity - that is, showing no effect.

\section{LEVELS OF RISK}

The figure shows how the concentrations of total cholesterol, high density lipoprotein cholesterol, and triglycerides were related to risk of major ischaemic heart disease. For each blood lipid men were ordered into five groups of equal size. The numbers of cases of ischaemic heart disease in each fifth were used to calculate the (unadjusted) yearly risk per 1000 men. Total cholesterol concentration had the steepest risk gradient: men in the top fifth $(\geqslant 7 \cdot 2 \mathrm{mmol} / \mathrm{l})$ had over three times the risk of men in the bottom fifth $(<5 \cdot 5$ $\mathrm{mmol} / \mathrm{l}$ ). For high density lipoprotein cholesterol concentration men in the bottom fifth $(<0.93 \mathrm{mmol} / \mathrm{l})$ had more than twice the risk of men in the top fifth $(\geqslant 1.33 \mathrm{mmol} / \mathrm{l})$. These two risk gradients were only slightly reduced after adjustment for one another and for the other non-lipid risk factors mentioned above (figure). For triglyceride concentrations men in the top fifth $(\geqslant 2.8 \mathrm{mmol} / \mathrm{l})$ had more than twice the risk of men in the bottom fifth $(<1.08 \mathrm{mmol} / \mathrm{l})$, but this was much lower and not significant after adjustment for total cholesterol concentration and non-lipid factors (relative odds $1 \cdot 3$ ). These relative odds fell to 0.8 after adjustment for high density lipoprotein cholesterol concentrations as well.

We combined the predictive properties of total cholesterol and high density lipoprotein cholesterol concentrations by using multiple logistic regression. The most predictive linear combination score was $(0.42 \times$ total cholesterol concentration $)-$ high density lipoprotein cholesterol concentration. Although high density lipoprotein cholesterol concentration had the larger coefficient $(-1.0$ compared with 0.42$)$, total cholesterol concentration had greater variability, its standard deviation being four times that of high density lipoprotein cholesterol concentration, so that total cholesterol concentration was the more important contributor to this combined lipid score. Men in the top fifth of this score had relative odds of 4.4 compared with those in the bottom fifth after adjustment for non-lipid risk factors (figure), which was a substantially steeper risk gradient than that for total cholesterol or high density lipoprotein cholesterol concentration alone. Others have used the ratio of high density lipoprotein cholesterol to total cholesterol concentration instead ${ }^{16}$ but we found that its predictive power was slightly weaker. In a multiple logistic model containing both linear and ratio scores the linear risk score was more significant.

\section{INTERACTIONS BETWEEN LIPIDS}

Table III shows the rates of major ischaemic heart disease events in the men classified into thirds of high density lipoprotein cholesterol concentration and total cholesterol concentration after adjustment for the other non-lipid risk factors mentioned above. The increases in risk associated with raised total cholesterol concentration and lowered high density lipoprotein cholesterol concentration seemed to operate independently of one another. Men in the highest category of risk (total cholesterol concentration $>6.7 \mathrm{mmol} / \mathrm{l}$ and high density lipoprotein cholesterol concentration $<1.015 \mathrm{mmol} / \mathrm{l}$ ) had nearly five times the risk of 
ischaemic heart disease of men in the lowest category of risk.

A similar cross classification for triglyceride and total cholesterol concentrations shows the risk gradient for total cholesterol concentration at each third of triglyceride concentrations. For each of the thirds of total cholesterol there was little evidence that triglyceride concentrations were associated with risk of ischaemic heart disease. Table III also shows the positive association between total cholesterol and triglyceride concentrations - for example, men in the top third of the distribution of triglyceride concentrations comprised $20 \%, 30 \%$, and $50 \%$ of those in the bottom, middle, and top thirds of total cholesterol concentration, respectively.

\section{PRE-EXISTING ISCHAEMIC HEART DISEASE}

Based on previous studies ${ }^{17}$ men were classified into three groups at the initial screening: group 1-
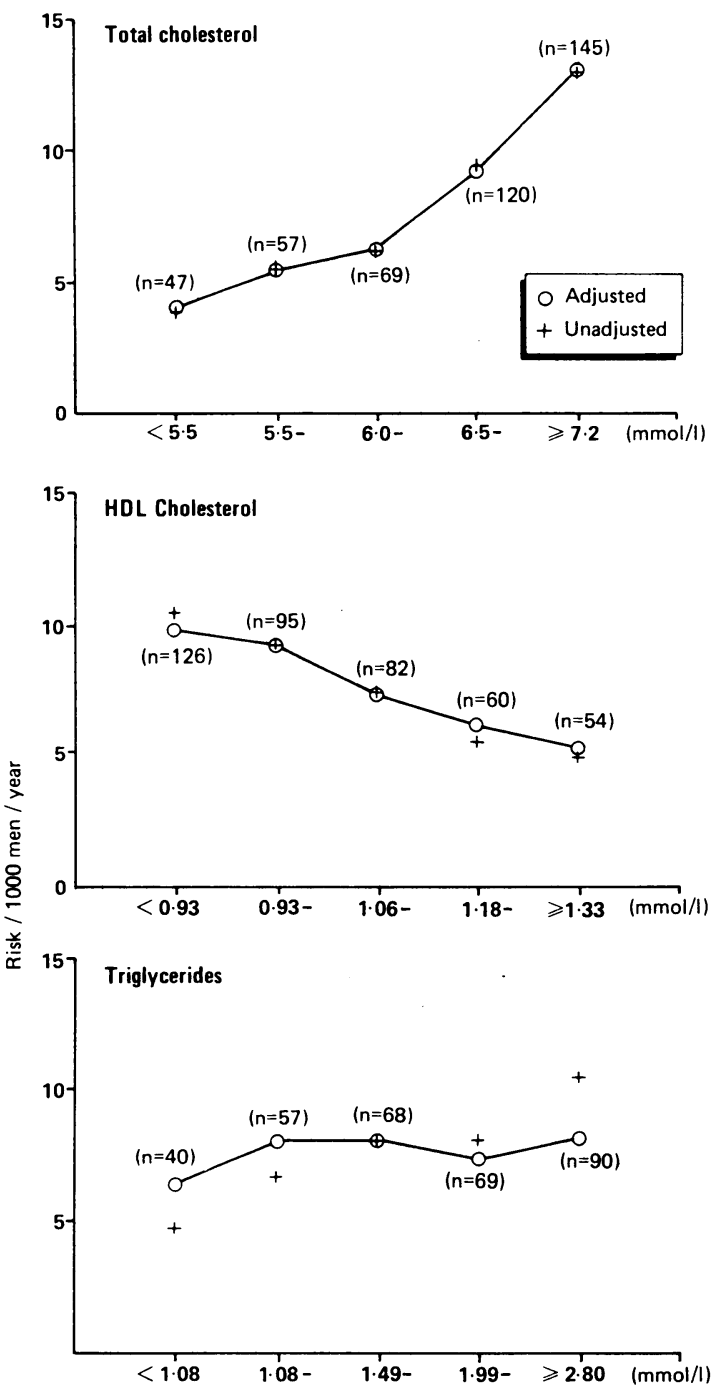

Risk of major ischaemic heart disease events $/ 1000$ men/year in each fifth of distribution of concentrations of serum total cholesterol, high density lipoprotein $(H D L)$ cholesterol triglycerides, and lipid score $((0.42 \times$ total cholesterol concentration) $-H D L$

cholesterol concentration).

Numbers of cases of ischaemic heart diseases in each fifth are shown in parentheses
TABLE III - Risk of major ischaemic heart disease events in each third of total cholesterol concentration by third of high density lipoprotein (HDL) and triglyceride concentrations. Figures are numbers of cases out of total number of men and adjusted yearly rate per 1000 men

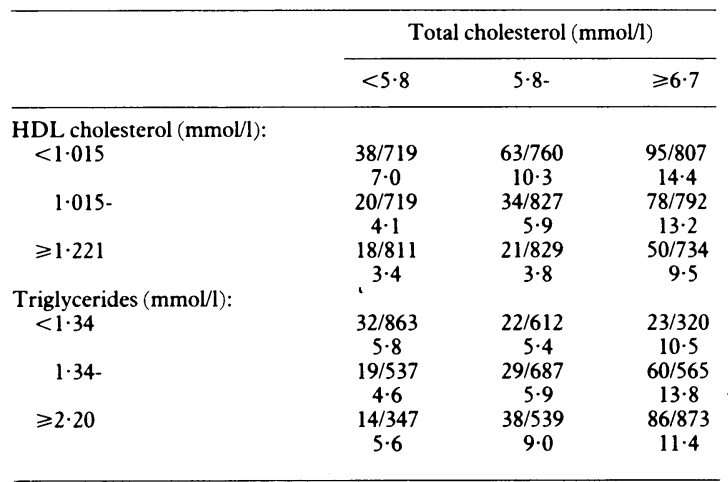

*Adjusted for age, systolic blood pressure, body mass index, and years of moking with a multiple logistic model.

TABLE IV-Mean serum concentrations of total cholesterol, high density lipoprotein $(H D L)$ cholesterol, and triglycerides in men who became cases of ischaemic heart disease (IHD) and other men classified into previous definite myocardial infarction (group 1), other evidence of IHD (group 2), and no evidence of IHD (group 3)

\begin{tabular}{|c|c|c|c|c|}
\hline & \multicolumn{2}{|c|}{$\begin{array}{l}\text { Mean concentration } \\
(\mathrm{mmol} / \mathrm{l})\end{array}$} & \multirow{2}{*}{\multicolumn{2}{|c|}{$\begin{array}{l}\text { Difference in means betweer } \\
\text { IHD cases and other men }\end{array}$}} \\
\hline & \multirow{2}{*}{$\begin{array}{l}\text { IHD } \\
\text { cases }\end{array}$} & \multirow{2}{*}{$\begin{array}{l}\text { Other } \\
\text { men }\end{array}$} & & \\
\hline & & & Unadjusted & Adjusted (SE) \\
\hline \multicolumn{5}{|c|}{ Total cholesterol: } \\
\hline Group 1 & $6 \cdot 814$ & $6 \cdot 494$ & $0 \cdot 320$ & $0 \cdot 290(0 \cdot 137)$ \\
\hline Group 2 & $6 \cdot 714$ & $6 \cdot 301$ & 0.413 & $0.407(0.099)$ \\
\hline Group 3 & $6 \cdot 764$ & $6 \cdot 251$ & 0.513 & $0.474(0.074)$ \\
\hline \multicolumn{5}{|c|}{ HDL cholesterol: } \\
\hline Group 1 & $1 \cdot 040$ & $1 \cdot 087$ & -0.047 & $-0.019(0.029)$ \\
\hline Group 2 & 1.065 & $1 \cdot 149$ & -0.084 & $-0.086(0.026)$ \\
\hline Group 3 & 1.096 & $1 \cdot 155$ & -0.059 & $-0.049(0.018)$ \\
\hline \multicolumn{5}{|c|}{ Triglycerides: } \\
\hline Group 1 & $2 \cdot 365$ & $2 \cdot 307$ & 0.058 & $-0 \cdot 180(0 \cdot 167)$ \\
\hline Group 2 & $2 \cdot 324$ & $2 \cdot 101$ & 0.223 & $-0.036(0.143)$ \\
\hline Group 3 & $2 \cdot 322$ & 1.997 & 0.325 & $0.065(0 \cdot 100)$ \\
\hline
\end{tabular}

previous definite myocardial infarction, according to an electrocardiogram or the patient's recall of the doctor's diagnosis $(n=428)$; group 2 -other evidence of ischaemic heart disease (that is, myocardial ischaemia according to an electrocardiogram, angina or possible myocardial infarction on the basis of a standardised questionnaire) or patient's recall of a doctor diagnosing angina $(n=1515)$; group $3-$ no evidence of ischaemic heart disease $(n=5767)$.

Table IV shows the mean difference for each blood lipid between cases and other men in each of these three groups. Total cholesterol concentration had a significant mean difference in all three groups even after adjustment for high density lipoprotein cholesterol concentration and non-lipid risk factors. A weaker inverse pattern was seen for high density lipoprotein cholesterol concentration, though for men with previous definite myocardial infarction the mean difference in high density lipoprotein cholesterol concentration between new cases and others was not significant. Mean triglyceride concentrations were higher in men without pre-existing disease who became cases; this was not of predictive significance after adjustment for other risk factors.

\section{Discussion}

HIGH DENSITY LIPOPROTEIN CHOLESTEROL

These results from the British regional heart study add substantially to current knowledge about high density lipoprotein cholesterol concentration and risk of ischaemic heart disease. We analysed over twice as many cases as in our previous report, a larger body of 
information than that in any other prospective study of high density lipoprotein cholesterol concentration. We adopted a different approach from that in our previous work in assessing how the ability of high density lipoprotein cholesterol concentration to predict ischaemic heart disease is affected by allowance for other risk factors. Adjustment for non-high density lipoprotein cholesterol concentration may be inappropriate ${ }^{5}$ as it includes both low density lipoprotein and very low density lipoprotein cholesterol concentrations. Low density lipoprotein cholesterol concentration is related to risk of ischaemic heart disease, whereas very low density lipoprotein cholesterol concentration is inversely associated with high density lipoprotein cholesterol concentration so that their combination falsely produces a confounding variable associated with both risk of ischaemic heart disease and high density lipoprotein cholesterol concentrations. Also, as non-high density lipoprotein cholesterol concentration was determined by subtracting the high densi ${ }^{*}$. lipoprotein cholesterol from total cholesterol , acentration rather than by independent measurement, variation within laboratories will have inflated the inverse association between concentrations of non-high density lipoprotein cholesterol and high density lipoprotein cholesterol. In the absence of direct measurement of low density lipoprotein cholesterol concentration it seems that adjustment for total cholesterol concentration (and other non-lipid risk factors) is more reliable in assessing the independent predictive role of high density lipoprotein cholesterol concentration. ${ }^{5}$ Also, our increased follow up resulted in a slightly increased (and more precise) estimate of the univariate association between high density lipoprotein cholesterol concentration and risk of major ischaemic heart disease.

We now see stronger evidence for a "protective effect" of high density lipoprotein cholesterol concentration on ischaemic heart disease than we previously reported. We would still not claim, however, that high density lipoprotein cholesterol concentration is a major risk factor as the risk gradients for the established risk factors (total cholesterol concentration, systolic blood pressure, and cigarette smoking) are all substantially steeper. ${ }^{11}$ In particular, it is more important to know a man's serum total cholesterol concentration than his high density lipoprotein cholesterol concentration when determining his risk of ischaemic heart disease.

Is measuring high density lipoprotein cholesterol concentration worth while in screening programmes for risk of ischaemic heart disease? Practical difficulties concern quality control within laboratories and the lack of facilities for routine determination of the concentration. Also, adding high density lipoprotein cholesterol concentration to assessments of multiple risk factors contributes little further discriminatory power. Nevertheless, if both total cholesterol and high density lipoprotein cholesterol concentrations are available it is worth using a combined lipid score. Whereas the ratio of high density lipoprotein cholesterol to total cholesterol concentration has been fashionable, a linear score $((0.42 \times$ total cholesterol concentration)-high density lipoprotein cholesterol concentration) provided better prediction with our data. Total cholesterol concentration is the more important contributor to such a combined score.

Quantitative comparisons with other studies are useful. From table II we estimated a $2 \cdot 5 \%$ reduction in risk of major ischaemic heart disease for every 0.026 $\mathrm{mmol} / \mathrm{l}(10 \mathrm{mg} / \mathrm{l})$ increase in high density lipoprotein cholesterol concentration ( $95 \%$ confidence interval 1.4 to $3 \cdot 7 \%$ ). A recent overview from the United States seems compatible, having corresponding percentage reductions of $2 \cdot 0 \%, 2 \cdot 0 \%$, and $2 \cdot 8 \%$ for men in the Framingham heart study, the multiple risk factor intervention trial, and the Lipid Research Clinics coronary prevention trial, respectively. ${ }^{5}$

Is high density lipoprotein cholesterol concentration of prognostic importance for men with pre-existing ischaemic heart disease? The Israeli ischaemic heart disease study reported that high density lipoprotein cholesterol and total cholesterol concentrations had similar predictive power for mortality in men with a previous myocardial infarction. ${ }^{18}$ We found that total cholesterol concentration was the more powerful predictor of subsequent major ischaemic heart disease (fatal and non-fatal) in men with definite myocardial infarction at initial screening.

\section{TRIGLYCERIDES}

Many prospective studies have shown that fasting serum triglyceride concentrations are positively associated with risk of ischaemic heart disease, but most of these studies have found that the concentrations are not an independent predictor of ischaemic heart disease after adjustment for other risk factors. ${ }^{19-25}$ Three studies have concluded that fasting triglyceride concentrations do have an independent role..$^{26-28}$ This lack of consistent evidence brings into question the justification for treating isolated moderate hypertriglyceridaemia.?

The relative merits of fasting and non-fasting blood samples for measuring triglyceride concentrations need careful consideration. As there is a considerable and rapid response in triglyceride concentration to dietary intake, particularly of saturated fat, use of nonfasting samples may give a better representation of the role of triglycerides in the relation between diet and ischaemic heart disease. In this report we have presented the first substantial prospective findings for non-fasting serum triglyceride concentrations, which are consistent with a previous review of fasting triglyceride concentrations ${ }^{7}$ - that is, moderate increases in non-fasting serum triglyceride concentrations are associated with an increased risk of ischaemic heart disease, but this can be accounted for by the association of triglyceride concentrations with total cholesterol and high density lipoprotein cholesterol concentrations, leaving non-fasting triglyceride concentrations of no value as an independent predictor.

Comparison of fasting and non-fasting concentrations of triglycerides in general population studies is of interest. A study based on general practices in four British towns showed that the overall distributions of fasting and non-fasting triglyceride concentrations were similar. ${ }^{29}$

Two studies have suggested that raised triglyceride concentrations may indicate increased risk in particular subsets of men. The Paris prospective study showed that serum triglyceride concentrations predicted risk of ischaemic heart disease in men with low serum concentrations of total cholesterol $(<220 \mathrm{mg} / 100 \mathrm{ml}$ $(0.572 \mathrm{mmol} / \mathrm{l})) .^{30}$ The Framingham study proposed that higher triglyceride concentrations are linked to increased risk if the ratio of total cholesterol to high density lipoprotein cholesterol concentration is over $3 \cdot 5 .^{31}$ These contradictory findings support the view that analysis of subgroups in studies with limited numbers of cases of ischaemic heart disease may generate misleading information and should be interpreted with caution. Our data show no particular subgroup (classified by total cholesterol or high density lipoprotein cholesterol concentrations or their ratio) for which triglyceride concentration is an important risk factor.

Although others have suggested that for patients with pre-existing ischaemic heart disease the subsequent prognosis is related to serum triglyceride concentration, ${ }^{32}$ we found no evidence for that concentration having a substantial association with risk in 
men with previous definite myocardial infarction or other pre-existing ischaemic heart disease. Instead, serum total cholesterol concentration seems a much better lipid measurement to combine with other risk indicators in identifying subjects with a high risk of subsequent major ischaemic heart disease.

\section{CONCLUSION}

Our prospective findings in a large representative sample of British middle aged men confirm the view that serum total cholesterol concentration is the most useful single lipid measurement in identifying patients at high risk of ischaemic heart disease. The inverse association of high density lipoprotein cholesterol concentration with risk was also confirmed but was of lesser predictive value. Once total cholesterol concentration has been measured the measurement of high density lipoprotein cholesterol concentration may be of marginal additional value in general population screening and in intervention programmes for risk of ischaemic heart disease. Serum triglyceride concentrations are not relevant to the prediction of ischaemic heart disease in the general male population once these two measurements have been taken into account. Widespread use of measurements of triglyceride concentration and drugs to lower that concentration may be without justification.

The British regional heart study is a British Heart Foundation Research Group and is also supported by the Medical Research Council and the Department of Health. Serum total cholesterol and high density lipoprotein cholesterol concentrations were determined in the Wolfson Research Laboratories, Birmingham (Professor T P Whitehead). Triglyceride concentrations were determined in the Royal Free Hospital School of Medicine, London, by Dr D G Cramp and Mrs Ila Patel.

1 Enger SC, Hjermann I, Foss OP, et al. High density lipoprotein cholestero and myocardial infarction and sudden death: a prospective case-control and myocardial infarction and sudden death: a prospective case-co

2 Goldbourt U, Medalie JH. High density lipoprotein cholesterol and incidence of coronary heart disease-the Israeli ischemic heart disease study. Am $\mathcal{F}$ Epidemiol 1979;109:296-308.

3 Miller NE, Thelle DS, Forde OH, Mjos OD. The Tromso heart study. High density lipoprotein cholesterol and coronary heart disease: a prospective case control study. Lancet 1977; i:965-8.

4 Gordon T, Castelli WP, Hjortland MC, et al. High density lipoprotein cholesterol as a protective factor against coronary heart disease. The Framingham study. Am $\mathcal{Y}$ Med 1977;62:707-14.

5 Gordon DJ, Probstfield JL, Garrison RJ, et al. High density lipoprotein cholesterol and cardiovascular disease: four prospective American studies. Circulation 1989;79:8-15.

6 Pocock SJ, Shaper AG, Phillips AN, Walker M, Whitehead TP. High density lipoprotein cholesterol is not a major risk factor for ischaemic heart disease in British men. Br Med f 1986;292:515-9.

7 Hulley SB, Rosenman RH, Bawol RD, Brand RJ. Epidemiology as a guide to clinical decisions. The association between triglyceride and coronary heart disease. $N$ Engl f Med 1980;302:1383-9.
8 Expert Panel. Report of the national cholesterol education program on detection, evaluation and treatment of high blood cholesterol in adults. Arch Intern Med 1988;148:36-69.

9 Study Group of the European Atherosclerosis Society. Strategies for the prevention of coronary heart disease: a policy statement of the European Atherosclerosis Society. Eur Heart f 1987;8:77-88.

10 Shaper AG, Pocock SJ, Walker M, Cohen NM, Wale CJ, Thomson AG. British regional heart study: cardiovascular risk factors in middle-aged men in 24 towns. Br Med F 1981;283:179-86.

11 Shaper AG, Pocock SJ, Walker M, Phillips AN, Whitehead TP, Macfarlane $\mathrm{PW}$. Risk factors for ischaemic heart disease: the prospective phase of the British regional heart study. I Epidemiol Community Health 1985;39: 197-209.

12 Walker M, Shaper AG. Follow-up of subjects in prospective studies based in general practice. $f R$ Coll Gen Pract 1984;34:365-70.

13 Thelle DS, Shaper AG, Whitehead TP, Bullock DG, Ashby D, Patel I. Blood lipids in middle-aged British men. Br Heart f 1983;49:205-13.

14 Pocock SJ, Ashby D, Shaper AG, Walker M, Broughton PMG. Diurnal variation in serum biochemical and haematological measurements. $\mathcal{f}$ Clin Pathol (in press)

15 Lee J. Covariance adjustment of rates based on the multiple logistic regression model. $\mathcal{f}$ Chronic Dis 1981;34:415-26.

16 Brunner D, Weisbort J, Meshulam N, et al. Relation of serum total cholesterol and high-density lipoprotein cholesterol percentage to the incidence of definite coronary events: twenty-vear follow-up of the Donolo-Tel Aviv prospective coronary artery disease study. Am f Cardiol 1987;59:1271-6.

17 Phillips AN, Shaper AG, Pocock SJ, Walker M, Macfarlane DW. The role of risk factors in heart attacks occurring in men with pre-existing ischaemic heart disease. Br Heart $\mathcal{F}$ 1988;60:404-10.

18 Goldbourt U, Cohen L, Neufeld HN. High density lipoprotein cholesterol: prognosis after myocardial infarction: the Israeli ischaemic heart disease study. Int f Epidemiol 1986;15:51-5.

19 Tibblin G, Wilhelmsen L, Werko L. Risk factors for myocardial infarction and death due to ischemic heart disease and other causes. Am $\mathrm{f}$ Cardiol 1975;35:514-22.

20 Rosenman RH, Brand RJ, Sholtz RI, et al. Multivariate prediction of coronary heart disease during 8.5 year follow-up in the western collaborative group study. Am $\mathcal{F}$ Cardiol 1976;37:903-10.

21 Robertson TL, Kato H, Gordon T, et al. Epidemiologic studies of coronary heart disease and stroke in Japanese men living in Japan. Hawaii and California. Am $\mathcal{F}$ Cardiol 1977;39:244-9.

22 Coronary Drug Project Research Group. Natural history of myocardial infarction in the coronary drug project: long term prognostic importance of serum lipid levels. Am $\mathcal{C}$ Cardiol 1978;42:489-98.

23 Kannel WB, Castelli WP, Gordon T. Cholesterol in the prediction of atherosclerotic disease: new perspectives based on the Framingham study. Ann Intern Med 1979;90:85-91.

24 Heyden S, Heiss G, Hames GC, et al. Fasting triglycerides as predictors of tota and CHD mortality in Evans County, Georgia. F Chronic Dis 1980;33:275 82 .

25 Yano K, Reed DM, McGee DL. Ten-year incidence of coronary heart disease in the Honolulu heart program: relationship to biologic and life-style characteristics. Am f Epidemiol 1984;119:653-66.

26 Carlson LA, Bottiger LE, Ahfeldt PE. Risk factors for myocardial infarction in the Stockholm prospective study: a 14-year follow-up focussing on the role the Stockholm prospective study: a 14-year follow-up focussing on the role

27 Pelkonen R, Nikkila EA, Koskinen S, et al. Association of serum lipids and obesity with cardiovascular mortality. Br Med f 1977;ii: 1185-7.

28 Petersson B. Trell E. Hood B. Premature death and associated risk factors in urban middle-aged men. Am $\mathcal{J}$ Med 1984;77:418-26.

29 Mann JI, Lewis B, Shepherd J, et al. Blood lipid concentrations and other cardiovascular risk factors: distribution, prevalence and detection in Britain. Br Med f 1988;296:1702-6.

30 Cambien F, Jacqueson A, Richard JL, et al. Is the level of serum triglyceride a significant predictor of coronary death in normocholesterolemic subjects? The Paris prospective study. Am f Epidemiol 1986;124:624-32.

31 Castelli WP. The triglyceride issue: a view from Framingham. Am Heart $f$ 1986;112:432-7.

32 Fox $\mathrm{MH}$, Gruchow HW, Barboriak JJ, et al. Risk factors among patients undergoing repeat aorta-coronary by-pass procedures. $\mathcal{I}$ Thorac Cardiovas Surg 1987;93:56-61.

(Accepted 13 February 1989)

\section{Department of Child \\ Health, St George's \\ Hospital Medical School, London SW17 0RE Richard West, MD, senior lecturer \\ Anne Davies, BA, research fellow \\ Timothy Fenton, MRCP, senior registrar}

Correspondence to: Dr West.

BrMed f 1989;298:1002-3

\section{Accidental vulval injuries in childhood}

\section{Richard West, Anne Davies, Timothy Fenton}

In girls the three most common types of accidental injury to the external genitalia are injuries caused by straddling, accidental penetration, and tearing due to sudden forced stretching of the perineum when the legs are abducted suddenly, such as can occur during gymnastics or in some falls. ${ }^{12}$ Accidental injury to the hymen is rare. ${ }^{3}$ Children with accidental injuries to the external genitalia should be referred to a paediatrician so that possible sexual abuse is not missed. Patients and families must be handled sensitively so that they do not think that they are under suspicion.

Accidental injury was diagnosed in 13 patients referred over the past three years for a paediatric opinion. Over the same period about 100 children who had probably or definitely been sexually abused were seen

Case 1-A would be gymnast lost her balance while walking along a horizontal metal pole and fell astride it. Her mother took her immediately to the family doctor, who referred her to a paediatrician. A complete and clear history was given. On examination there was a linear bruise $2 \mathrm{~cm}$ long on the inner surface of the left labium minus anteriorly, along the line of the underlying pubic ramus. The injury matched the history, and accidental injury was diagnosed.

The table summarises the 13 cases of accidental injury seen.

\section{Comment}

In straddle accidents injury is due to forced compression of soft tissues between the object straddled 